Daimon. Revista Internacional de Filosofía, $n^{\circ} 75$ (Septiembre-Diciembre) 2018, 27-41

ISSN: 1130-0507 (papel) y 1989-4651 (electrónico)

http://dx.doi.org/10.6018/daimon/332161

\title{
Disposiciones y puntos de vista causales*
}

\author{
Dispositions and causal points of view
}

\begin{abstract}
Resumen: Este artículo trata principalmente de la importancia ontológica de las propiedades disposicionales y, en defensa de su eficacia causal, subraya las diferencias entre dos puntos de vista sobre la causalidad, el propio de la causalidad eficiente, que es el habitual, y el correspondiente a la causalidad estructural, concluyendo que las disposiciones se identifican con causas estructurales. Sin embargo, aunque ambos puntos de vista sobre la causalidad son solo distintos y se complementan, surgen significativos conflictos entre ellos.

Palabras clave: disposiciones, puntos de vista, causalidad eficiente, causalidad estructural.
\end{abstract}

SEBASTIÁN ÁLVAREZ TOLEDO**

\section{Introducción}

\begin{abstract}
This paper is mainly about the ontological signification of dispositional properties, and in order to uphold the causal efficacy of dispositions, it highlights the differences between two points of view on causation: that of efficient causation, which is the usual one, and that corresponding to structural causation. The conclusion is that dispositions are structural causes. However, although both points of view on causation are just different and complement each other, some meaningful conflicts arise between them.

Keywords: dispositions, points of view, efficient causation, structural causation.
\end{abstract}

Entre diferentes puntos de vista sobre un mismo asunto caben relaciones muy distintas $\mathrm{y}$, en consecuencia, también son distintos los motivos para optar por uno u otro. Puede que se trate de puntos de vista incompatibles pero comparables, como, por ejemplo, el creacionismo y el evolucionismo, y en tales casos existen o, al menos, son posibles criterios bien fundados e intersubjetivos para tomar una decisión. Sin embargo, puede ocurrir que los puntos de vista además de incompatibles sean incomparables entre sí, como lo serían, por ejemplo, los paradigmas alternativos en una etapa de crisis, según el primer Kuhn, o los distintos estilos o tendencias históricos o actuales en el arte. En estas circunstancias la opción por uno u otro deberá nutrirse de elementos ajenos al objeto de los puntos de

Recibido: 24/05/2018. Aceptado: 21/10/2018.

* Este artículo es resultado del Proyecto de investigación FFI2014-57409. Points of View, Dispositions and Time. Perspectives in a World of Dispositions (Ministerio de Economía y Competitividad, Gobierno de España).

** Profesor jubilado de la Facultad de Filosofía de la Universidad de Salamanca. Email: sat@usal.es. Líneas de investigación: Causalidad; tiempo; leyes de la naturaleza; clases naturales; unidad y diversidad en la ciencia. Algunas publicaciones recientes: "Causation and the agent's point of view", Theoria , 29, 1, (2013), pp. 133147; "Kinds, Laws and Perspectives”, en: M. Vázquez and M. Liz (eds.): Point of View and Temporal Structures, Heidelberg: Springer, 2015, pp. 235-253. 
vista en sí mismo, es decir, será subjetiva o sustancialmente dependiente de determinado contexto social y cultural. Siendo así, es inevitable ser relativista en el análisis y valoración de tal diversidad de puntos de vista y de opciones. Pero, sobre un mismo asunto pueden existir también diferentes puntos de vista compatibles y, en mayor o menor medida, complementarios. Por ejemplo, se puede abordar el estudio de una película atendiendo específicamente al guión, la dirección, la interpretación, la banda sonora, etc.; del mismo modo que podemos tomar fotografías del Duomo de Florencia desde diferentes lugares de su entorno. En ambos casos cada punto de vista aporta una información adicional que permite un mejor conocimiento de la película o del Duomo. La pluralidad de puntos de vista de este tipo se debe al carácter polifacético de la realidad, y la incorporación de uno nuevo contribuye a la configuración de una imagen más rica en detalles o en perspectivas del objeto en cuestión. Esta confluencia de puntos de vista no exige elegir entre ellos como en los casos anteriores, ni tiene sentido ser relativista ante la existencia de tal diversidad. La actitud más adecuada ante ella es el perspectivismo, basado en que la realidad es esencialmente múltiple y nuestro acceso a ella es siempre parcial y dependiente de forma inevitable de uno o varios puntos de vista ${ }^{1}$. Pero, como veremos indirectamente en este artículo, no por ello hay que suponer que la existencia de puntos de vista compatibles y complementarios y la actitud perspectivista remitan necesariamente a una confluencia armónica y carente de conflictos de estos.

Aunque no sea su tema central, el presente artículo incorpora como base de su argumentación un análisis de las relaciones entre dos puntos de vista concretos cuyas conclusiones pueden resultar interesantes para una concepción general de los puntos de vista. El artículo trata principalmente de la importancia de las propiedades disposicionales en ontología y de algunas de las críticas al respecto, en particular, de los argumentos contra la eficacia causal de las disposiciones. Mi respuesta a tales argumentos se basa en una revisión del concepto de causalidad en la que, insistiendo en la naturaleza pluricausal de los efectos, reivindico el punto de vista internalista, relativo a las causas estructurales, frente a la tradicional hegemonía del punto de vista externalista, propio de la causalidad eficiente. A partir de ahí se puede ver cómo la caracterización de las causas estructurales coincide perfectamente con la de las disposiciones.

Sin embargo, aunque los dos puntos de vista causales mencionados son compatibles y complementarios, el hecho de reclamar la debida atención para las causas estructurales no se traduce sencillamente en la incorporación de un punto de vista que viene a enriquecer con nuevos aspectos nuestra comprensión de las relaciones causales, sino que, debido a la habitual preeminencia del punto de vista externalista, la inclusión de este otro suscita reticencias y malentendidos, exige revisar las prerrogativas concedidas a la causalidad eficiente, logra desarticular críticas tradicionales al concepto de causalidad y, en suma, conduce a un cambio sustancial en la concepción general de las relaciones causales.

1 Sobre una concepción general del perspectivismo y sus diferencias tanto con el relativismo como con el absolutismo puede consultarse Liz (2013), especialmente apartados 3 ,4 y 7, y Liz and Vázquez (2015), especialmente apartados 3,4 y 5 . 


\section{El interés ontológico de las disposiciones}

Es tradicional en filosofía una distinción entre propiedades de las cosas, según la cual, existen, por una parte, las propiedades llamadas categóricas, que son siempre manifiestas, esto es, no dependen para su manifestación de que se den determinadas condiciones; por ejemplo, la altura de un edificio, la forma de un jarrón o la composición química de una sustancia. Y por otra parte, existen propiedades que solo se manifiestan, o tienden a hacerlo, en determinadas condiciones, ante determinados estímulos, como ocurre con la fragilidad de un jarrón, la elasticidad de una cuerda de guitarra o la toxicidad de una sustancia. El comportamiento de estas propiedades, llamadas disposicionales, o simplemente disposiciones, es representable, por tanto, mediante un condicional cuyo antecedente sería el estímulo o estímulos necesarios y el consiguiente, su manifestación. Un cuerpo es frágil si al golpearlo se rompe, una cuerda es elástica si recupera su forma cuando cesa la fuerza que la ha deformado, y una sustancia es tóxica si cuando la ingerimos enfermamos ${ }^{2}$. Las disposiciones reciben en la literatura filosófica nombres diferentes, como capacidades, tendencias, poderes o propensiones (aunque no siempre estos términos puedan considerarse sinónimos).

Las propiedades disposicionales han suscitado algunas sospechas en la filosofía de orientación analítica por su falta de observación directa y se ha intentado mantenerlas al margen de la ontología fundamental (por ejemplo, Carnap, 1936 o Armstrong, 1996), sin embargo, es notable el interés que este tipo de propiedades ha adquirido en las últimas décadas, debido a motivos de distintos tipos. Por una parte, y como veremos, un análisis mínimamente atento muestra que el ámbito de las propiedades disposicionales es mucho más extenso de lo que podría parecer, hasta el punto de que, como sostiene Mumford (2011), es difícil encontrar una propiedad que no tenga un aspecto disposicional. Tengamos en cuenta, por ejemplo, que el estado líquido del agua o la solidez de un trozo de hierro dependen de la temperatura del medio en que se encuentren o que el acero a temperaturas extremadamente bajas es frágil. Por otra parte, muchos autores han visto en el concepto de disposición u otros equivalentes un elemento fundamental para afrontar distintas cuestiones ontológicas en filosofía de la ciencia. Recordemos, por ejemplo, la importancia que concedía Popper a la idea de propensión en la imagen científica del mundo. En apoyo de su concepción indeterminista de la naturaleza, defendía una teoría de la probabilidad como propensión, según la cual, las probabilidades son tendencias de los sucesos a generar determinados resultados, que así explican la frecuencia relativa de estos en diferentes experimentos: como fuerzas que mantienen la estabilidad de las estadísticas. Esta concepción de las probabilidades es para Popper un nuevo punto de vista de "los procesos que constituyen nuestro mundo", un punto de vista contrario a la "ideología determinista". Vivimos -repite en muchos de sus trabajos- en "un mundo de propensiones", un mundo siempre abierto a nuevas posibilidades (Popper, 1990, chap. 1).

No menos enfática es la defensa que hace Cartwright (1989) de las capacidades de la naturaleza. Sostiene que las leyes causales, fundamentales en la investigación científica, solo son posibles porque existen capacidades naturales, elementos insustituibles en la ontología

2 De todos modos, una disposición se puede manifestar de formas diferentes: la disposición a hablar francés se puede manifestar hablando, escribiendo, etc. (Bird 2007, 21) 
de la ciencia. Como es sabido, la formulación de una ley causal es fruto de una labor de abstracción o idealización, de modo que en su formulación final dichas leyes se refieren a modelos altamente contrafácticos, están sujetas en su aplicación a un gran número de excepciones y cuando se cumplen, no lo hacen de forma exacta sino sólo aproximadamente. Según Cartwright, todo ello significa que a lo que realmente se refieren las leyes causales no es a sucesos o regularidades de nuestra experiencia directa, sino a capacidades, en cuanto patrones flexibles de comportamiento: "the logic that uses what happens in ideal circumstances to explain what happens in real ones is the logic of tendencies or capacities". Dicho de otra forma, la capacidades se sitúan en un nivel de modalidad superior al de las leyes causales y refuerzan nuestra confianza en la estabilidad y regularidad de estas. Por todo ello las capacidades naturales forman parte inevitablemente de nuestra imagen científica del mundo (pp. 1, 147 y 190).

De modo similar defiende Chakravartty (2013) que los conceptos de capacidad, disposición, propensión o tendencia, en los que se basa actualmente la idea de causalidad, son necesarios para explicar las regularidades que las leyes de la naturaleza describen. Y para Mumford (2013), solo una ontología de disposiciones puede romper con la insatisfactoria idea de una naturaleza muerta, pasiva, sin vida, gobernada por leyes como elementos dinamizadores.

Dedicaré el siguiente apartado a analizar brevemente algunas discusiones en torno a la importancia ontológica de las disposiciones, sin embargo, creo que antes de seguir es oportuno desarticular una ambigüedad que encontramos continuamente en el uso del concepto de disposición.

Se puede entender una disposición como la capacidad de un sistema para actuar sobre otro modificándolo. Así cuando decimos que el café es excitante o que el agua es un gran disolvente estamos atribuyendo a estas sustancias la capacidad de modificar otros cuerpos, que acusarían su influencia, su acción. Otros adjetivos disposicionales de este tipo serían, por ejemplo: fertilizante, propulsor, condensador, tóxico, letal, legible, indignante, estresante, adelgazante, agradable, apetecible, etc. Pero también se suele entender una disposición como la capacidad de reacción de un sistema ante cambios que ocurren en su entorno. Así cuando decimos que una persona es muy irritable o que una sustancia es soluble, seguimos hablando de disposiciones o capacidades (irritabilidad, solubilidad), pero nos hemos situado al otro lado de la relación, en el sistema en que tiene lugar la manifestación. Adjetivos disposicionales de esta clase serían también, por ejemplo: frágil, elástico, flexible, combustible, sumergible, condensable, coagulable, dilatable, irascible, crédulo, etc. Tanto en el lenguaje cotidiano como en el filosófico sobre disposiciones se mezclan esta dos acepciones y en la mayoría de los casos carece de especial interés distinguir entre una y otra: expresiones como que el calor tienen la capacidad de dilatar los metales y que los metales tienen la capacidad de dilatarse con el calor se pueden considerar equivalentes en muchos contextos. Sin embargo, desde un perspectiva ontológica, el asunto es algo diferente, porque se trata de dos enfoques redundantes: la capacidad dilatadora del calor y la de ser dilatable de los metales no es que se complementen, es que se solapan innecesariamente, y por mera cuestión de simplicidad parece conveniente unificar el significado del término disposición optando por uno de los enfoques. En este asunto creo que es más adecuado optar por el segundo enfoque, es decir, definir las disposiciones como 
capacidades de reacción de los sistemas en determinadas condiciones o ante determinados estímulos. Aunque se trata de una opción en cierta medida convencional, encontramos una buena razón a su favor en las disposiciones atribuidas a sustancias o situaciones cuyas manifestaciones tienen lugar en los seres humanos. Qué duda cabe de que disposiciones como ser tóxico, letal, potable, digerible, alimenticio, confortable, sabroso, irritante, inteligible, etc. muestran un claro carácter antropocéntrico: nada tiene de suyo ninguna de estas propiedades, y al atribuírselas nos estamos refiriendo indirecta o figuradamente a determinadas capacidades de reacción nuestras, biológicas y mentales. A partir de ahí creo que, en general, no es nada artificial considerar que términos disposicionales como disolvente, condensador o dilatador constituyen también formas metafóricas de referirse a capacidades reactivas de ciertos sistemas, como ser soluble, condensable o dilatable.

\section{Objeciones a las propiedades disposicionales}

Las discusiones acerca de la importancia ontológica del concepto de disposición presentan diferentes frentes. Me centraré en dos de ellos: el que se refiere a las relaciones entre propiedades categóricas y disposicionales y el que trata de la relevancia causal de las disposiciones.

Hay quienes niegan que las disposiciones sean realmente propiedades. Según Armstrong, por ejemplo, las genuinas propiedades, como es el caso de las propiedades categóricas, son autoconsistentes, permanentes y para describirlas no es necesario hacer referencia a algo más allá de ellas mismas, como son los comportamientos o efectos adicionales que puedan generar; las disposiciones, por el contrario, serían solo formas abreviadas de referirse a propiedades categóricas atendiendo a sus comportamientos. Dicho de otro modo, toda pretendida propiedad disposicional remite inevitablemente a una base categórica, que es la que hace verdadero al condicional implícito en la adscripción de una disposición. Así, por ejemplo, decir que un objeto es frágil no consiste en atribuirle una propiedad real sino en afirmar que, debido a su estructura cristalina, que sí es una propiedad genuina, se romperá cuando se golpea. En consecuencia, las supuestas propiedades disposicionales son analizables o reducibles a propiedades categóricas (Armstrong, 1968, pp. 85-89; 1997, pp. 79-80). Cerca de Armstrong en esta cuestión se sitúan Quine (1974) y Mackie (1977).

Sin embargo, los partidarios de la existencia de propiedades disposicionales responden que si bien estas tienen un carácter condicional, lo cierto es que lo condicionado es solo su manifestación, no la disposición en sí misma. Un cristal se rompe si se golpea, pero su fragilidad no depende del golpe, existe aunque nunca se manifieste: un objeto es frágil aunque nunca se golpee ni se rompa (Bird, 2007, pp. 66-67). Por otra parte, hay razones para pensar que gran parte, al menos, de las propiedades que se podrían calificar como categóricas presentan un innegable sesgo disposicional, es decir, se manifiestan condicionalmente. ¿Cómo una propiedad no va a introducir una diferencia causal en el mundo? En este sentido son posiblemente disposicionales predicados como blando u opaco, o actitudes como confianza, creencia, afecto, etc. (Mumford, 1998, p. v). En suma, no parece que resulte satisfactoria la definición de una propiedad sin atender a sus virtualidades nómicas o causales, esto es, al comportamiento del sistema a que se atribuye. No es de extrañar 
que argumentos de este tipo hayan conducido a muchos filósofos a adoptar un monismo disposicional, es decir, la conclusión de que, en el fondo, toda propiedad es esencialmente una disposición. Es el caso, por ejemplo, de Popper, Haré, Madden, Shoemaker, Mumford, Bird, Chakravartty o Whittle. Con menos radicalidad otros autores admiten la existencia de algunas propiedades categóricas, como es el caso de Swoyer, Ellis o Molnar, aunque se refieren tan solo a ciertas propiedades geométricas o espaciotemporales, con lo cual las propiedades categóricas quedarían reducidas a características como la triangularidad, la esfericidad o existir en determinadas fechas, tener tal edad, etc. (Cf. Choi and Fara, 2016, paragraph 3).

Pasemos a la discusión acerca de la relevancia causal de las disposiciones. Como acabamos de ver, la realidad de las propiedades disposicionales está vinculada a la de sus capacidades causales. Sin embargo, hay un largo debate acerca de este punto. McKitrick (2005) señala dos típicos argumentos contra la relevancia causal de las disposiciones: el argumento de analiticidad y el de exclusión. El primero consiste básicamente en exigir el requisito humeano de independencia lógica entre las descripciones de la causa y del efecto (causa y efecto han de ser existencias distintas) y advierte que si el significado de una disposición consiste en su tendencia a producir una determinada manifestación en las condiciones adecuadas, la conexión entre la disposición y su manifestación carece de la contingencia propia de la relación entre causa y efecto. Según esta objeción, no cabe decir que la causa de la ruptura del vaso fue su fragilidad o que la fusión de un metal se debió a que era fusible. Como se suele decir, estas atribuciones de causalidad tendrían el mismo aire tautológico que la explicación que leemos en El enfermo imaginario de Molière de que opio adormece porque tiene virtus dormitiva. Encontramos este tipo de objeción, por ejemplo, en Mackie, Armstrong, Block o Jackson. (Cf. Choi and Fara, 2016, paragraph 6).

Sin embargo, Mumford (1998, pp. 140-142) no cree que esta objeción sea realmente importante. Admite que en multitud de casos las disposiciones no ofrecen explicaciones de sus manifestaciones, pero esto se debe a que el éxito de una explicación depende en gran medida de las circunstancias en que esta se produce (entre ellas, los conocimientos de los destinatarios), y no todas las causas que aparecen en un explanandum resultan igualmente informativas. Es cierto, si buscamos la explicación del incendio que se ha producido en una vivienda, de poco vale que nos indiquen que había oxígeno en ella, aunque no cabe duda de que el oxígeno fue una de las causas de ese incendio. Según Mumford, en la mencionada objeción de analiticidad, se da una confusión entre lo explicativo y lo ontológico que conduce a tomar la escasa utilidad explicativa de las disposiciones como síntoma o consecuencia de su falta de eficacia causal. Por tanto, se puede decir que las disposiciones causan sus manifestaciones aun en los casos en que no las expliquen causalmente.

De modo parecido responde McKitrick (2005) a este argumento de analiticidad advirtiendo que en él se confunden lo semántico y lo metafísico. Se refiere a que el aparente aspecto tautológico o analítico de la atribución de causalidad a las disposiciones se debe sencillamente a que, por lo general, los nombres de estas (extensible, frágil, fusible, etc.) aluden directamente a sus manifestaciones, por lo que no es de esperar que, si nos atenemos al significado etimológico de sus nombres, tales atribuciones digan algo nuevo. Por eso advierte que no debemos confundir las relaciones entre nuestros conceptos con las relaciones entre cosas en el mundo y que encontrar una relación trivial entre conceptos 
no excluye encontrar una relación interesante entre "las entidades que caen bajo esos conceptos". Según esta respuesta, la forma de eludir la acusación de analiticidad consistirá en acercar lo semántico y lo metafísico enriqueciendo el significado de los términos disposicionales, esto es, pasando de su significado meramente etimológico a su contenido real, de forma que, por ejemplo, la elasticidad de un cuerpo remita a la organización y fuerza de cohesión de sus moléculas. De este modo desaparece la analiticidad porque el término disposicional ya no se refiere exclusivamente a su manifestación.

Pero llegados a este punto, nos encontramos con una nueva objeción, la que McKitrick llama argumento de exclusión, según el cual, la pretendida eficacia causal de las disposiciones no reside en ellas mismas sino en su base causal: si una disposiciones se debe a la composición y estructura del sistema, como la elasticidad respecto a la cohesión molecular, la disposición no tiene ninguna eficacia o relevancia causal propia. Sin embargo, a esta objeción se puede responder que si bien es verdad que, como hemos visto, las propiedades disposicionales son susceptibles de análisis en términos de elementos y estructuras más básicos, eso mismo se puede decir de toda propiedad en general, ya sea disposicional o categórica. En gran medida el progreso del conocimiento consiste en el descubrimiento de explicaciones más profundas de propiedades y fenómenos conocidos. Por tanto, aunque no es difícil admitir que no existen disposiciones "desnudas", es decir, sin una base de nivel inferior ${ }^{3}$, la cuestión ahora es si esta base habría de ser una propiedad categórica o, por el contrario, podría seguir siendo de carácter disposicional. Y es preciso advertir además que aquí la cuestión no es siquiera si determinadas disposiciones tienen una base causal categórica, sino si toda disposición debe tenerla. Porque si no fuera así, habría disposiciones con eficacia causal. Obviamente, quienes, como Armstrong o Prior, defienden que las propiedades fundamentales han de ser categóricas sostendrán que la aparente eficacia causal de las disposiciones se debe a propiedades categóricas básicas; una opción nada fácil de sostener si, como hemos visto, el ámbito de las propiedades genuinamente categórica parece alarmantemente reducido

Mencionaré, por último otras dos objeciones a la eficacia causal de las disposiciones. Cabe objetar que lo habitual es considerar que las causas son sucesos o cambios pero no propiedades o estados de cosa. Así, por ejemplo, se entiende que la causa de la herida no fue propiamente la masa o la forma de la piedra sino su impacto en el cuerpo, la pedrada. Por esta misma razón y dado que las disposiciones son propiedades, no sucesos, no cabe decir que puedan ser causas de sus manifestaciones. Como dice Cummins (1974), atribuir una disposición a un sistema equivale a decir que reúne una serie de condiciones tales que sucesos de cierto tipo pueden causar en él la manifestación de tal disposición, lo que implica que la disposición no es realmente la causa. Y muy relacionada con esta objeción está la que presenta García Encinas (2011), según la cual, una disposición no puede ser causa de sus manifestaciones porque, de ser así, causa y efecto pertenecerían a la misma entidad, lo que equivaldría a confundir la relación causal con una actividad interna, con un proceso interno de un sistema. Según esta objeción, en la disolución de un grano de sal

3 No obstante, autores como Ellis, Molnar, McKitrick y Mumford argumentan que, dado que partículas subatómicas fundamentales como leptones y quarks carecen de partes constituyentes y de estructura, sus propiedades disposicionales carecerían de una base causal, serían disposiciones "desnudas" (Cf. Choi and Fara, 2016, paragraph 4). Pero creo que podemos prescindir aquí de este argumento. 
en agua la causa no es la solubilidad de ese grano, sino la acción del agua sobre él. Esta objeción conecta con el tradicional requisito de exterioridad de la causa. Comentaré estas dos objeciones más adelante, al final del siguiente apartado, que está dedicado a un análisis de la noción de causalidad, en cuyo marco adquiere pleno sentido el carácter causal de las disposiciones.

\section{Puntos de vista causales}

Es habitual definir la relación causal como una relación irreflexiva, asimétrica y transitiva entre dos sucesos, la causa y el efecto. La irreflexividad comporta tanto una independencia lógica entre los conceptos de causa y efecto (la no analiticidad antes mencionada) como la distinción entre los sistemas relacionados causalmente, lo que a su vez supone el referido requisito de exterioridad de la causa respecto al sistema en que ocurre el efecto. Todo ello se cumple, por ejemplo, en el caso de la antorcha que ocasiona el incendio de una cabaña. Sin embargo, esta definición es excesivamente esquemática, porque todo efecto es pluricausal, ningún suceso es efecto de una sola causa, sino de la confluencia de toda una serie de sucesos y circunstancias favorables (lo que Stuart Mill llamó la "causa total"). En el ejemplo anterior la cabaña no habría ardido si además de la antorcha no hubiese en el entorno oxígeno y algo de viento, si estuviera lloviendo torrencialmente, si la cabaña no fuera de madera o de algún material combustible, etc. Mackie (1980, chap. 3), que es tal vez el autor que más ha insistido recientemente en el carácter pluricausal de las relaciones causales, define las causas como condiciones INUS. Parte del hecho de que, por lo general, un efecto $\mathrm{P}$ puede haber sido producido de modos diferentes, es decir, puede deberse a diferentes conjuntos de causas, a causas totales alternativas, ABC, DGH, JKL, que, no obstante, pueden tener elementos comunes. Por tanto, la disyunción ABC o DGH o JKL representa la condición necesaria y suficiente para que suceda $\mathrm{P}$, y cualquier miembro, cualquier causa total, de esta disyunción, digamos $\mathrm{ABC}$, representa una condición suficiente pero no necesaria para $\mathrm{P}$, una condición además suficiente mínima, dado que ninguno de sus elementos es redundante ni suficiente para P. Siendo así, un elemento cualquiera de una condición suficiente mínima, pongamos A, no es necesario ni suficiente para P. Lo que quiere decir, por ejemplo, que acercar una cerilla a un objeto no es necesario ni suficiente para que este arda. Sin embargo, ese elemento está relacionado con $\mathrm{P}$ de un modo importante: es un elemento insuficiente pero necesario de una causa total, es decir, de una condición no necesaria pero suficiente para P. Por esto Mackie llama a los elementos de una causa total de un efecto singular P condiciones INUS de P (tomando las iniciales de Insufficient, Necessary, Unnecessary y Sufficient).

Es fácil suponer además que en la cuidadosa enumeración de las distintas causas o condiciones INUS de un efecto concreto figurarán algunas que no son externas, sino propias del sistema en que ocurre el efecto, como la combustibilidad de la madera de la cabaña en nuestro ejemplo anterior. También si enumeramos las distintas causas del crecimiento de una planta tendremos que incluir, junto a factores externos como la luz solar, el agua de la tierra, etc., la capacidad de la propia planta para generar materia orgánica mediante fotosíntesis. La importancia de los factores causales internos en la relación causal nos recuerda inevitablemente al modelo de causalidad aristotélico, en el que, junto a las cau- 
sas externa o eficientes, figuran las causas que él llamó materiales y formales, que son debidas a la estructura o naturaleza del sistema en que ocurre el efecto. Por consiguiente, la definición de causalidad que he esbozado al comienzo de este apartado, y que es la más común, se correspondería solo con un punto de vista parcial de la causalidad: el de la causalidad eficiente.

En términos generales, hay que entender la causalidad interna como una reacción de un sistema ante cambios que se producen en su entorno. Cuando alguien gritar en la calle el nombre de una persona y esta se vuelve o cuando calentamos un trozo de metal y se dilata o se funde se trata de reacciones de esa persona o ese trozo de metal ante sucesos o cambios a su alrededor. Más concretamente, este tipo de reacciones consiste en el desarrollo de una serie de cambios, en un proceso, a veces de una gran complejidad, cuyo resultado es el efecto. En el caso de la fusión del metal lo que ocurre es que al ponerlo en contacto con una fuente de calor intenso sus moléculas incrementan su agitación y se debilita la fuerza de atracción entre ellas hasta el punto de que resbalan unas sobre otras y el cuerpo pierde su estado sólido. En los seres vivos este tipo de reacciones internas ante los cambios en su medio responden típicamente a la propiedad de homeostasis, esto es, la tendencia a mantener un determinado equilibrio entre los valores de ciertas variables básicas. Ejemplos de homeostasis son la termorregulación, que en los humanos permite mantener el organismo a una temperatura en torno a los $36,5^{\circ} \mathrm{C}$., o el proceso de cicatrización de una herida. Cabría incluso utilizar el concepto de homeostasis, aunque no en un sentido estricto, referido a los procesos que constituyen la causalidad interna en general, no sólo en biología. Así, por ejemplo, no es forzado calificar de homeostáticos a los cambios que se producen en los valores de la presión, la temperatura y el volumen de un gas cuando cambian las condiciones externas, para recuperar un equilibrio entre estas variables ${ }^{4}$.

Estas consideraciones acerca de la causalidad interna o estructural se corresponden con un punto de vista de la causalidad diferente en varios aspectos al más habitual, que es, sin duda alguna, el punto de vista que podemos llamar externalista, centrado en las causas eficientes. No se trata, claro está, de puntos de vista incompatibles, porque cualquier efecto surge de causas de uno y otro tipo. Resaltar la importancia de la actividad interna de los sistemas en la generación o producción de los efectos no implica vindicar ningún tipo de autosuficiencia por parte de estos. Los sistemas no son mónadas y la causalidad sigue manteniendo su tradicional carácter contrafáctico, según el cual, si nada cambia en el exterior de un sistema no se produce en este ningún cambio que podamos llamar efecto. Tanto las causas eficientes como las estructurales son condiciones INUS (como las llama Mackie) para la producción de un efecto. Por tanto, la causalidad eficiente y la estructural constituyen dos puntos de vista significativamente distintos, pero no sólo compatibles, sino además complementarios.

Sin embargo, son puntos de vista que enfatizan aspectos muy diferentes de las relaciones causales. Si atendemos a la importancia y complejidad de los procesos que constituyen la causalidad interna en una relación causal, no cabe ya concebir la causalidad, como es tan habitual, como la actividad productiva de uno de sus elementos (la causa o causas eficientes)

4 Cf. Álvarez Toledo (2014). Boyd (1991; 1999) utiliza esta idea de homeostasis en sentido amplio en su definición de las clases naturales como "racimos homeostáticos de propiedades". 
sobre un sistema meramente pasivo, en el que se produciría el efecto. Tendemos a pensar que el hecho de regar una planta mustia produce su revitalización, pero lo que en realidad ocurre es que la planta, al recuperar mediante sus raíces el agua de que carecía, reactiva la fotosíntesis, es decir, vuelve a metabolizar, gracias a la energía solar, el dióxido de carbono del aire y a producir los hidratos de carbono que necesita para seguir viviendo. De modo que las supuestas capacidad productiva y actividad del riego para resucitar o mantener la vida de una planta quedan notablemente atenuadas, si no pasan a ser meramente metafóricas. Cabe decir incluso que, desde el punto de vista internalista de la causalidad pierde sentido el viejo concepto de "poder causal" asociado a la causalidad eficiente: no es que el riego o el agua tenga el poder de reavivar la planta sino que esta, cuando detecta agua en su entorno, dirige hacia ella sus raíces, la absorbe y reinicia la compleja actividad que conocemos como fotosíntesis ${ }^{5}$.

En este sentido, defiende Joy (2013) que la conocida oposición de Hume a la realidad de las relaciones causales no es sino un rechazo a la idea de poder causal que se suele atribuir a las causas eficientes. La definición de causalidad que Hume contempla incluye, como es sabido, la continuidad espacial entre causa y efecto, que supone la exterioridad de la causa, y la prioridad temporal de esta respecto del efecto, como ocurre, por ejemplo, en el choque de dos bolas de billar que tiene como efecto el inicio del movimiento de una de ellas ${ }^{6}$; unos rasgos que responden claramente a la causalidad eficiente. Y su crítica se centra en la imposibilidad de descubrir racionalmente en la causa ninguna propiedad que haga necesaria la producción del efecto; de ahí que defienda que causa y efecto son sucesos regularmente consecutivos, pero desconectados ${ }^{7}$, y que carece de justificación atribuir a las causas el poder de producir sus efectos.

Sin embargo, sostiene Joy que esta recusación de la causalidad tiene como complemento en Hume la creencia de que existen en los objetos ciertas capacidades o propensiones a los cambios, cuya activación genera los fenómenos que llamamos efectos. A favor de esta hipótesis interpretativa Joy presenta un "razonamiento metafísico de estilo humeano" en el que se dice: (a) que todo objeto que cambia tiene previamente una capacidad para ese cambio; (b) que tal capacidad no es suficiente para que este se produzca y se necesita la contribución de un objeto o suceso distinto; (c) que por esta razón ese objeto o suceso va constantemente unido al cambio resultante, es decir, al efecto. Añade Joy que en este razonamiento se sobreentiende que la relación entre una de estas capacidades ${ }^{8}$ y el cambio correspondiente no es en sí misma una relación causal, lo que coincide con la anterior apreciación de que, para Hume, causalidad es solo causalidad eficiente. Y Joy aplica esta interpretación de Hume a la versión de este de los principios de la física newtoniana. Antes de Hume algunos filó-

5 De todos modos, el término de "poder causal" es utilizado a veces con un significado más amplio y se aplica tanto a las causas eficientes como a las estructurales o internas (Por ejemplo, Cartwright and Pemberton, 2013). Pero aquí me limito a su uso más habitual como atributo de las causas eficientes.

6 Hume, 1739-1740, book 1, part 3, sect. 2 and 6.

7 "The mind can never possibly find the effect in the supposed cause, by the most accurate scrutiny and examination. For the effect is totally different from the cause, and consequently can never be discovered in it" (Hume, 1772, 4. 9).

8 Joy llama a estas capacidades "disposiciones causales extrínsecas" para indicar que su realización o manifestación necesita de unas condiciones externas favorables. 
sofos modernos habían intentado rehabilitar la idea aristotélica y medieval de poder causal aplicándola a las fuerzas de la física newtoniana, consideradas principios activos, causas de los movimientos y los cambios de la materia, que de suyo sería pasiva. Hume, sin embargo, entiende que no hay que interpretar tales fuerzas como poderes causales intrínsecos a un objeto material o como capacidades de influencia de un cuerpo sobre otro, sino, según la interpretación de Joy, como impulsos generados en este segundo cuerpo en respuesta a una colisión, que modifican su estado de reposo o de movimiento uniforme. Y dado que se considera que las leyes del movimiento de Newton son fundamentales en la mecánica clásica, podemos atribuir este tipo de capacidades o disposiciones a todo objeto material. Añade Joy que la presunta presencia de este tipo de disposiciones en la ontología de Hume le permitía admitir un "principio restringido de uniformidad de la naturaleza" que, sin llegar a desarticular su escepticismo respecto a la inducción, justificaría la confianza en muchas de nuestras predicciones.

Pero continuemos con las diferencias entre los dos puntos de vista causales. El punto de vista externalista, propio de la causalidad eficiente, al atender tan solo a la correlación entre la acción de un factor externo sobre un sistema y el cambio que ocurre en este, responde básicamente a un esquema de caja negra, mientras que el punto de vista internalista, en el que lo importante es la complicada serie de cambios con que el sistema reacciona a modificaciones ocurridas en su entorno, responde a un esquema de caja translúcida. Si tomamos como ejemplo el movimiento de un girasol durante el día, la descripción desde el punto de vista de la causalidad eficiente señalaría a la luz solar con sus distintas posiciones relativas como la causa del giro de la planta, sin atender, como haría el punto de vista internalista, a la compleja actividad de las hormonas responsables de su fototropismo, mediante el cual el girasol se adapta al giro de la Tierra de modo que los rayos del sol sigan cayendo sobre sus pétalos. No cabe duda de que esta actividad interna es más importante biológicamente que el seguimiento del giro de la flor. En general, los procesos internos que se corresponden con la causalidad estructural son cognitivamente más relevantes que los sucesos externos que les sirven de detonantes. En este punto es oportuno recordar las frecuentes críticas a la noción de causalidad (eficiente) basadas en la convicción de que la física moderna puede prescindir perfectamente de ella. Por ejemplo, según Mach (1883, p. 580), aunque se dice que el calor es la causa de la presión de un gas, cuando llegamos a conocer bien el fenómeno prescindimos de la causa y pensamos en el gas con la presión propia de su temperatura. Dicho de otro modo, desde el punto de vista de la física teórica, es más interesante conocer el modo en que se correlacionan interiormente la presión, la temperatura y el volumen en un gas que atender a los sucesos externos ante los que tiene lugar una reorganización de los valores de estas propiedades ${ }^{9}$. Y no muy distinta era la postura de Russell (1913) cuando advertía que la noción de causalidad es innecesaria en la ciencia moderna y que perderá su interés cuando los filósofos se familiaricen con la idea matemática de función, más adecuada que la de causalidad (eficiente) para representar las correlaciones entre valores de las propiedades de los sistemas. Esta referencia a Mach y a Russell tiene por objeto subrayar el interés cognitivo

9 En uno de sus conocidos aforismos, el físico español recientemente desaparecido Jorge Wagensberg afirmaba: "La ciencia no trata del porqué de las cosas, sino del cómo" (2006, p. 84). El libro donde aparece este aforismo se titula significativamente A más cómo, menos por qué. 
y científico de los procesos internos de los sistemas frente a la tradicional hegemonía de la causalidad eficiente, pero no creo en modo alguno que estos autores acertaran al certificar la defunción del concepto de causalidad eficiente en la ciencia.

Llegados a este punto, parece inevitable preguntarse por las razones que explican la hegemonía de la causalidad eficiente en los últimos siglos. Me limitaré a apuntar las dos que creo más importantes. Por una parte, en sus comienzos y en gran medida luego, la ciencia moderna ha estado vinculada a una concepción mecanicista del mundo, y parece evidente que en los fenómenos mecánicos la actividad de los sistemas en los que se produce un efecto (pensemos en la bola que empieza a moverse tras un choque) es muy elemental, casi irrelevante, en comparación con la complejidad de procesos internos químicos, biológicos, psicológicos o sociales. Por otra parte, es obvio que desde un punto de vista pragmático, la causalidad eficiente permite ser concebida como mera relación entre medios y fines, esto es, como un dispositivo mediante el cual podemos obtener (o evitar) determinados resultados realizando determinadas acciones, sin que tengamos que ocuparnos de los pasos intermedios que los conectan. De hecho, resulta poco discutible la hipótesis de que en la formación o adquisición del concepto de causalidad influye notablemente nuestra condición de agentes intencionales en la naturaleza; condición que nos inclina a interpretar las relaciones causales como correlaciones entre estímulos y respuestas en las que carece de importancia la actividad del sistema que responde. En conexión con este carácter selectivo de la noción de causalidad como causalidad eficiente, es oportuno recordar, por último, que, como subraya Mackie, en nuestro uso de los conceptos causales, la mención de una sola causa de entre las muchas que constituyen la causa total de un efecto obedece a criterios que varían según las circunstancias, pero se da en nuestro lenguaje causal una tendencia a señalar como la causa al elemento que en determinado contexto resulta raro, externo, "intruso", y no a lo que ocurre dentro del sistema en que tiene lugar el efecto. Ciertamente, diríamos que la causa de una hemorragia fue el corte con el cuchillo pero no el bombeo de la sangre por el corazón, aunque sea necesario para la hemorragia (Mackie 1980, pp. 31-57).

\section{Las disposiciones como causas y un apunte sobre puntos de vista}

Tras estas consideraciones acerca de los puntos de vista causales y la importancia de las causas estructurales parece obvio que las disposiciones, en cuanto capacidades de reacción de los sistemas ante cambios en su entorno, son causas de sus manifestaciones, es decir, forman parte de la causa total de ellas. Esta es la opinión de, por ejemplo, Chakravartti (2013), quien, convencido de que la causalidad se explica en última instancia por las disposiciones asociadas a ciertas entidades por las que estas exhiben determinados comportamientos, concluye que "disposición es un concepto causal por excelencia". Por su parte, Mumford y Anjum (2011, chap. 5 and 8), advierten de los perjuicios que para nuestra forma de pensar sobre la causalidad supone el "modelo de dos sucesos" (es decir, la hegemonía de la causalidad eficiente) e insisten en los vínculos causales existentes en los "procesos en los que una cosa se convierte gradualmente en otra" (pp. 113-116), como ocurre, por ejemplo, cuando el azúcar se disuelve en agua o la temperatura de una habitación en la que hay una estufa se va incrementando hasta alcanzar cierto límite. De ahí la excepcional importancia de las disposiciones como fundamento de las relaciones causales. 
Pero no es menos obvio que se trata de causas internas o estructurales, no de causas eficientes. Esta advertencia es importante para responder a dos impugnaciones mencionadas anteriormente a la relevancia causal de las disposiciones. Según vimos, decía Cummins que, si, como se entiende habitualmente, las causas son sucesos, no propiedades, las disposiciones no pueden ser causas, aunque se trate de propiedades que permiten que determinados sucesos externos causen ciertos efectos en las cosas. Esta objeción parte del supuesto erróneo de que las causas han de ser siempre sucesos. Lo son en el caso de la causalidad eficiente: como veíamos en un ejemplo anterior, entendemos que la causa de una herida puede ser una pedrada, que es un suceso, y no la masa o la forma de la piedra. Pero en el caso de la causalidad estructural es distinto, porque lo que se exige del sistema en que ocurre el efecto (la condición INUS correspondiente) es precisamente que esté dotado de una determinada propiedad disposicional. Similar a esta objeción es la también mencionada de García Encinas que negaba el carácter causal de las disposiciones partiendo de las premisas de que las causas suceden en el entorno de los sistemas en los que ocurre el efecto, pero no en ellos, y de que, por tanto, no existe relación causal entre las propiedades o sucesos que forman parte de la actividad interna de los sistemas. También en este caso la objeción solo tiene sentido si pensamos en la causalidad como causalidad eficiente.

Por último, creo oportuno señalar que, por cuanto hemos podido ver en este artículo, el argumento de analiticidad comentado anteriormente no constituye una objeción a la eficacia causal de las disposiciones. No es simplemente que un trozo de metal se funda porque es fusible o un vaso se rompa porque es frágil, sino que tanto la fusibilidad como la fragilidad nos remiten a las reacciones y procesos internos que constituyen el aspecto estructural de la causalidad.

Como indicaba al principio, este artículo se desarrolla sobre el trasfondo de la relación entre dos puntos de vista sobre la causalidad compatibles y complementarios pero significativamente distintos, y las conclusiones obtenidas sobre tal relación pueden ser útiles, como datos del estudio de un caso, en la elaboración de un panorama general de los puntos de vista y las relaciones entre ellos. La coexistencia de puntos de vista compatibles y complementarios sobre un mismo asunto y el perspectivismo como actitud ante tal diversidad pueden suscitar la idea, porque suele ser así, de una convivencia pacífica entre puntos de vista en la que cada uno de ellos se limita a aportar una perspectiva parcial del asunto en cuestión contribuyendo a enriquecer la comprensión de este. Pero no siempre es así, como muestran las relaciones entre los puntos de vista causales mencionados en este artículo. Las diferencias de perspectivas entre ellos junto al hecho de que uno de esos puntos de vista, el de causalidad eficiente, haya venido gozando de cierta supremacía respecto al otro, hasta el punto de que se haya llegado a una drástica reducción del concepto de causalidad, identificado generalmente como causalidad eficiente, han originado una serie de malentendidos, confusiones y críticas al concepto mismo de causalidad. En tal circunstancia el hecho de subrayar la importancia de las causas estructurales no se reduce a sumar otro punto de vista, sino que, además de hacer hincapié en el carácter pluricausal de los fenómenos, pone de manifiesto las deficiencias de lo que Mumford y Anjum llaman el "modelo de dos sucesos", obliga a revisar los conceptos de actividad y poder causal tradicionalmente vinculados a las causas eficientes, acentúa el interés cognitivo, dentro y fuera de la ciencia, de las relaciones causales y permite responder de un modo eficaz a críticas tradicionales a la causalidad, desarticulando los malentendidos 
de los que parten. En resumen, la vindicación de las causas internas o estructurales como rehabilitación de un punto de vista indebidamente relegado en la práctica no se limita a enriquecer la imagen general del objeto, en este caso la causalidad, añadiendo una nueva perspectiva parcial, sino que obliga a introducir cambios sustanciales en tal imagen.

\section{Referencias}

Álvarez Toledo, S. (2014), «Causation and the Agent's Point of View», Theoria, 79, pp. 133-147.

Armstrong, D. M. (1968), A Materialist Theory of Mind, rev. edn., 1993, London: Routledge.

Armstrong, D. M. (1996), «Categoricalist Versus Dispositionalist Accounts of Properties», Acta Analytica, 15, pp. 7-19.

Armstrong, D. M. (1997), A World of States of Affairs, Cambridge: Cambridge University Press.

Bird, A. (2007), Nature's Metaphysics, Oxford: Clarendon Press.

Boyd, R. (1991), «Realism, Anti-Foundationism and the Enthusiasm for Natural Kinds», Philosophical Studies, 61, pp. 127-148.

Boyd, R. (1999), «Homeostasis, species and higher taxes», in: R. A. Wilson (ed.): Species. New Interdisciplinary Essays, Cambridge, Mass.: MIT Press, pp. 142-185.

Carnap, R. (1936), «Testability and Meaning», Part I, Philosophy of Science, 3, pp. 420-471.

Cartwright, N. (1989), Nature's Capacities and their Measurements, Oxford: Oxford University Press.

Cartwright, N. and Pemberton, J. (2013), «Aristotelian Powers. Without Them, What Would Modern Science do?», in: R. Groff and J. Greco (eds.), (2013), pp. 93-112.

Chakravartty, A. (2013), «Dispositions for Scientific Realism», in: R. Groff and J. Greco (eds.) (2013), pp. 113-126.

Choi, S. and Fara, M. (2016), «Dispositions», The Stanford Encyclopedia of Philosophy (Spring 2016 Edition), Edward N. Zalta (ed.), URL = <https://plato.stanford.edu/archives/spr2016/entries/dispositions/>.

Cummins, R. (1974), «Dispositions, States and Causes», Analysis, 34, pp. 194-204.

García Encinas, M. J. (2011), «Singular Causation Without Disposition», Theoria, 26:70, pp. 35-50.

Groff, R. and Greco, J (eds.) (2013), Powers and Capacities in Philosophy. The New Aristotelism, New York: Routledge.

Hume, D. (1739-1740), Treatise on Human Nature. D. F. Norton and M. J. Norton (eds.) Oxford: Oxford University Press, 2000.

Hume, D. (1772), An Enquiry Concerning Human Understanding. T. Beauchamp (ed.) Oxford: Oxford University Press, 1999.

Joy, L. (2013), «The Ineliminability of Dispositions in Hume's Rejection of Causal Powers», in: R. Groff and J. Greco (eds.) (2013), pp. 69-90.

Liz, M. (2013), «Analizando la noción de puntos de vista», en: M. Liz (ed.): Puntos de vista. Una investigación filosófica, Barcelona: Laertes, pp. 25-164.

Liz, M. and Vázquez, M. (2015), «Subjective and Objective Aspects of Points of View», in: M. Vázquez and M. Liz (eds.): Temporal Points of View, Heidelberg: Springer, 2015, pp. 59-104. 
Mach, E. (1883), Die Mechanik in Ihrer Entwicklung Historisch-Kritisch Dargestellt. Leipzig: Brockhaus. English translation: The Science of Mechanics. La Salle: Open Court, 1960.

Mackie, J. L. (1977), «Dispositions, Grounds and Causes», Synthese, 34, pp. 361-369.

Mackie, J. L. (1980), The Cement of Universe, Oxford: Clarendon Press.

McKitrick, J. (2005), «Are Dispositions Causally Relevant?», Synthese, 144, pp. 357-371.

Mumford, S. (1998), Dispositions, Oxford: Oxford University Press.

Mumford, S. (2011), «Dispositions», Routledge Encyclopedia of Philosophy: www.rep. routledge.com/articles/thematic/dispositions/v-2.

Mumford, S. (2013), «The Power of Power», in: R. Groff and J. Greco (eds.) (2013), pp. 9-26.

Mumford, S. and Anjum, R. (1911,) Getting Causes from Powers, Oxford: Oxford University Press.

Popper, K. (1990), A World of Propensities, Bristol: Thoemmes.

Quine, W. V. O. (1974), Roots of Reference, La Salle, Ill.: Open Court.

Russell, B. (1913), «On the Notion of Cause», Proceeding of the Aristotelian Society, 13, pp. 1-26.

Wagensberg, J. (2006), A más cómo, menos por qué, Barcelona: Tusquets. 
